\title{
Microwave assisted synthesis of symmetrically and asymmetrically substituted acyclic enediynes
}

\author{
Mukesh Chandra Joshi, Penny Joshi, and Diwan S. Rawat* \\ Department of Chemistry, University of Delhi, Delhi-110007 India \\ E-mail: dsrawat@,chemistry.du.ac.in
}

\begin{abstract}
A convenient method for the synthesis of symmetrically and asymmetrically substituted acyclic enediynes has been developed using microwave irradiation. The reaction completes in 3-5 minutes, while same reaction takes $14-18$ h to complete under conventional heating method.
\end{abstract}

Keywords: Enediyne, Bergman cyclization, Sonogashira coupling, anti-cancer activity, antibacterial activity

\section{Introduction}

Enediyne chemistry has been subject of intense study in recent years due to its applications in medicinal and pharmaceutical chemistry. ${ }^{1-3}$ The anti-cancer activity of these compounds is due to the presence of highly unsaturated 1,5-diyne-3-ene unit that undergoes Bergman cyclization and generates benzene-1,4-diradical, which cleaves the DNA. ${ }^{4-7}$ Although enediynes possess potent anti-tumor activity but their clinical use has been limited because of their modest selectivity for cancer cells. Apart from anti-cancer activity, these compounds exhibit cytotoxicity against various cell lines, ${ }^{8,9}$ protein degradation activity, ${ }^{10}$ topoisomerase inhibitory activity ${ }^{11}$ and natural product enediynes are known to exhibit potent antibacterial activity against both grampositive and gram-negative strains. ${ }^{12}$ In order to improve the biological activity of the enediynes, efforts are being made to synthesize analogous compounds with better efficacy. ${ }^{13-19}$

Sonogashira coupling has been one of the reactions extensively used in the synthesis of enediyne framework. ${ }^{20-22}$ Lot of work has been done recently in the development of Sonogashira coupling and one of the development was the use of microwaves in order to promote the C-C bond formation. ${ }^{23-25}$ Microwave assisted synthesis has gained popularity in recent years, as it not only reduces the reaction time from hours to minutes, but also increases the yields. ${ }^{26}$ To the best of our knowledge there is no report of enediyne synthesis under microwave irradiation conditions. To this end, herein we report microwave assisted synthesis of symmetrically and asymmetrically substituted acyclic enediynes. 


\section{Results and Discussion}

The starting alkynes (1-8) used in the synthesis of acyclic enediynes, were prepared by literature or modified literature methods. Commercially available alcohols/phenols/thiophenol were treated with propargyl bromide in the presence of anhydrous $\mathrm{K}_{2} \mathrm{CO}_{3}$ in dry DMF or $\mathrm{NaH}$ in dry THF. The reaction was stirred at room temperature and progress of the reaction was monitored by TLC. The reaction takes $8-10 \mathrm{~h}$ to complete. After usual work up of the reaction mixture, the crude product was purified over silica gel column (scheme 1). The yield of the substituted alkynes ranged from $65-85 \%$. These alkynes (1-8) were used in the preparation of symmetrical or asymmetrical enediynes.

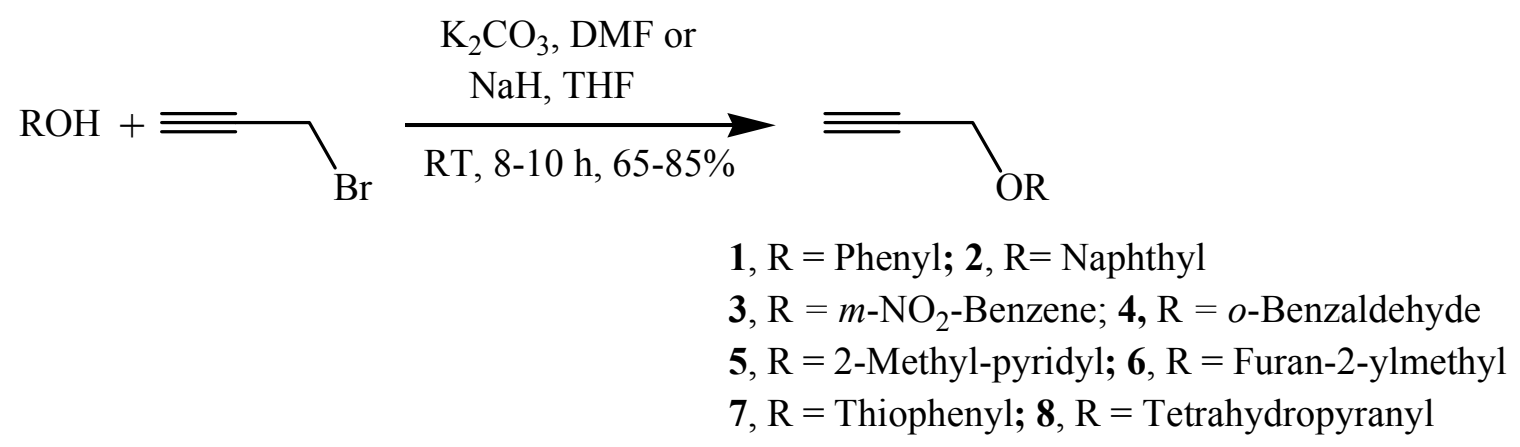

\section{Scheme 1}

Scheme 2 illustrates the synthesis of chloro-en-yne precursor $(\mathbf{9}, \mathbf{1 0})$ and symmetrical enediynes $(\mathbf{1 1}, \mathbf{1 2})$. A typical procedure for the synthesis of chloro-en-yne precursors $(\mathbf{9}, \mathbf{1 0})$, involves microwave irradiation $(650 \mathrm{~W})$ of a screw caped reaction vessel containing mixture of cis-dichloroethylene, substituted alkynes (1 equivalent each), catalytic amount of $\mathrm{Pd}\left(\mathrm{PPh}_{3}\right)_{4}, \mathrm{CuI}$ and $n-\mathrm{BuNH}_{2}$ in benzene. The progress of the reaction was monitored by TLC. Reaction completes in 3-5 minutes and crude product was purified by silica gel chromatography. The isolated yields of the chloro-en-yne precursor $(9,10)$ were ranged from $60-62 \%$ along with the symmetrical enediynes $(\mathbf{1 1}, \mathbf{1 2})$ in $9-13 \%$ yield.

Compounds 9 and $\mathbf{1 1}$ were earlier prepared in $70 \%$ and $40 \%$ yield respectively using copper acetylides and chloro-iodo-ethylene as starting materials. No analytical data was given for these compounds. ${ }^{27}$ When 0.5 equivalent of cis-dichloroethylene was reacted with 1.0 equivalent of substituted alkynes under identical reaction conditions as discussed above, 61-65\% of the symmetrical enediynes $(\mathbf{1 1}, \mathbf{1 2})$ were obtained along with $6-8 \%$ of the chloro-en-yne precursor $(9,10)$. Enediyne 12 was earlier prepared in $59 \%$ yield using conventional methodology and reaction completed in $16 \mathrm{~h}^{28}$ Asymmetrical enediynes were synthesized in good yields as the procedure described above and shown in scheme 3. 


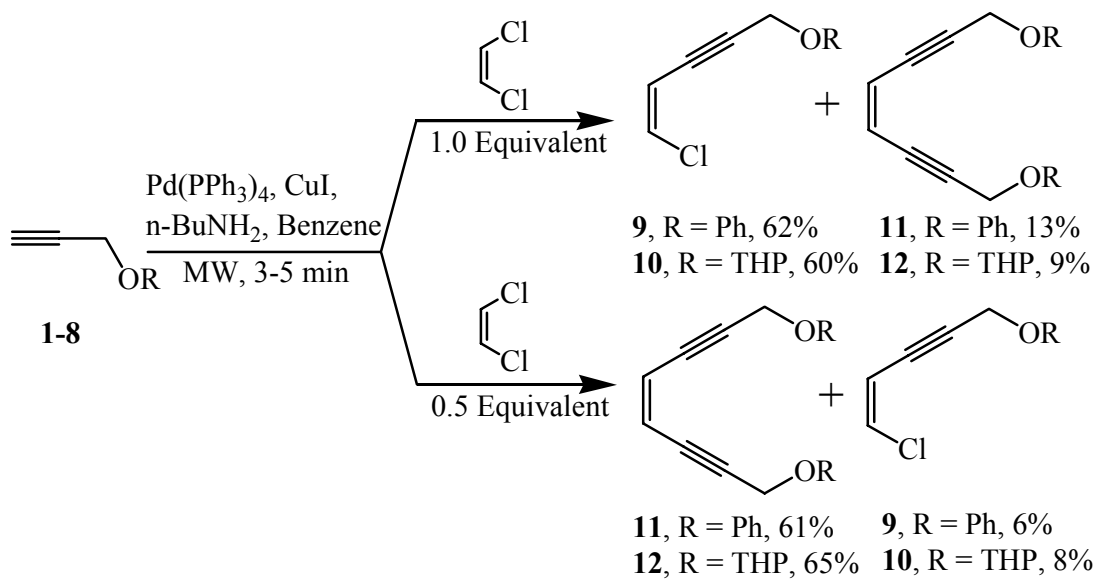

\section{Scheme 2}

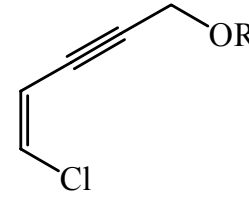

9, $\mathrm{R}=\mathrm{Ph}$

10, $\mathrm{R}=\mathrm{THP}$

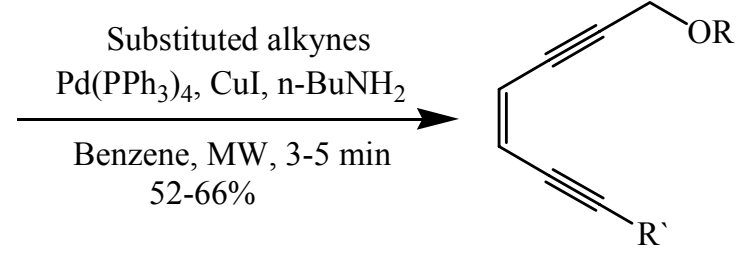

13-24

\section{Scheme 3}

For example, coupling of chloro-pent-4-en-2-ynyloxy-tetrahydro-pyran $(\mathbf{1 0})^{29}$ with prop-2ynyloxy-pyran (8) under microwave irradiations as discussed above, leads to the formation of the enediyne 14 in $62 \%$ yield in 3 minutes (scheme 3, table 1). Reaction of 2,3dibromothiophene (25) with 2-methyl-5-prop-2-ynyloxy-pyridine (5) or 2-prop-2-ynyloxytetrahydropyran (8) in the presence of catalytic amount of $\mathrm{Pd}\left(\mathrm{PPh}_{3}\right)_{4}, \mathrm{CuI}$ and $n-\mathrm{BuNH}_{2}$ in benzene under microwave irradiations, gave the desired enediynes $(\mathbf{2 6}, \mathbf{2 7})$ in $52-54 \%$ yield in 3 5 minutes (scheme 4).

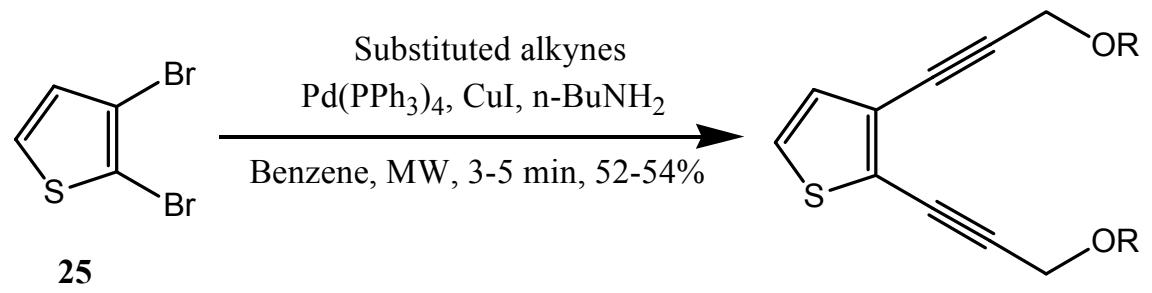

26, $\mathrm{R}=$ THP

27, $\mathrm{R}=$ 2-Methyl-pyridyl

\section{Scheme 4}


Table 1. Symmetrical and asymmetrical enediynes

Comp. No. Yield (\%) $\begin{aligned} & \text { Time } \\ & \text { (min.) }\end{aligned}$

While under conventional heating conditions this reaction takes $16-18 \mathrm{~h}$ to complete at $80{ }^{\circ} \mathrm{C}$. This reaction further confirms the utility of microwave irradiation in the enediyne synthesis. Microwave irradiation of $(\mathbf{1 3}, \mathbf{1 4})$ in presence of 100 equivalent of cyclohexadiene in benzene at $850 \mathrm{~W}$ for 30 minutes, results no cyclization of the enediynes. This clearly indicates that these compounds are stable under experimental conditions. Thermal cyclization temperature of these 
enediyes were measured on a neat materials by Differential scanning calorimetry (DSC). DSC clearly indicates an irreversible exothermic peaks at elevated temperatures, and results are given in the experimental section.

In summary, we have successfully developed a simple and efficient methodology for the synthesis of asymmetrically and symmetrically substituted acyclic enediynes using microwave irradiations. These reaction conditions provide an efficient method for the rapid access of medicinally important class of organic compounds.

\section{Experimental Section}

General Procedures. All of the chemicals used in the synthesis were purchased from SigmaAldrich and were used as such. Thin layer chromatography was used to monitor the progress of the reactions. All of the compounds were purified over silica gel column. Solvents were distilled before using for purification purposes. Melting points were determined on a melting point apparatus and are uncorrected. IR(KBr) spectra were recorded using Perkin-Elmer FT-IR spectrophotometer and the values are expressed as $v_{\max } \mathrm{cm}^{-1}$. Mass spectral data were recorded on a Jeol (Japan) JMS-DX303 and micromass LCT, Mass Spectrometer/Data system. The ${ }^{1} \mathrm{H}$ NMR and ${ }^{13} \mathrm{C}$ NMR spectra were recorded on Bruker Spectrospin spectrometer at $300 \mathrm{MHz}$ and 75.5 MHz, respectively using TMS as an internal standard. The chemical shift values are recorded on $\delta$ scale and the coupling constants $(J)$ are in Hz. Elemental analysis for all compounds were performed on a Carlo Erba Model EA-1108 elemental analyzer and data of C, $\mathrm{H}$ and $\mathrm{N}$ is within $\pm 0.4 \%$ of calculated values. Microwave reactions were performed in a domestic microwave oven of 850W (Kenstar model no: OM2ODGQX). Differential scanning calorimetry (DSC) traces were recorded on a Pyris 6 Differential scanning calorimeter of Perkin Elmer Corporation at a heating rate of $10{ }^{\circ} \mathrm{C} \mathrm{min}^{-1}$.

Synthesis of prop-2-ynyloxy-benzene ${ }^{30}$ (1). Yield: $4.76 \mathrm{gm}(85 \%)$; IR (KBr, cm$\left.{ }^{-1}\right)$ : 2922, 2867, 2122, 1597, 1494, 1455, 1374, 1215, 1174, 1036; ${ }^{1} \mathrm{H}$ NMR $\left(300 \mathrm{MHz}, \mathrm{CDCl}_{3}\right): 2.61(\mathrm{t}, \mathrm{J}=2.4$ $\mathrm{Hz}, 1 \mathrm{H}), 4.80(\mathrm{~d}, \mathrm{~J}=2.4 \mathrm{~Hz}, 2 \mathrm{H}), 7.15-7.20(\mathrm{~m}, 3 \mathrm{H}), 7.27-7.30(\mathrm{~m}, 2 \mathrm{H}) ; \mathrm{MS}(\mathrm{m} / \mathrm{z}): 132\left[\mathrm{M}^{+}\right]$; Anal. calcd. for $\mathrm{C}_{9} \mathrm{H}_{8} \mathrm{O}: \mathrm{C}, 81.79 ; \mathrm{H}, 6.10$. Found: $\mathrm{C}, 81.95 ; \mathrm{H}, 6.21$.

Synthesis of 2-prop-2-ynyloxy-naphthalene (2). Yield: 78\%; IR (KBr, cm $\left.{ }^{-1}\right) ; 2925,2218$, 1629, 1509, 1462, 1268, 1212, 1174, 1013; ${ }^{1} \mathrm{H}$ NMR $\left(300 \mathrm{MHz}, \mathrm{CDCl}_{3}\right): 2.65$ (t, J = $2.4 \mathrm{~Hz}$, $1 \mathrm{H}), 4.91(\mathrm{~d}, \mathrm{~J}=2.4 \mathrm{~Hz}, 2 \mathrm{H}), 7.23-7.38(\mathrm{~m}, 5 \mathrm{H}), 7.45-7.61(\mathrm{~m}, 2 \mathrm{H}) ; \mathrm{MS}(\mathrm{m} / \mathrm{z}): 182\left[\mathrm{M}^{+}\right]$.

Synthesis of 1-nitro-4-prop-2-ynyloxy-benzene (3). Yield: 85\%; mp 116-117 ${ }^{\circ} \mathrm{C}$ (lit. ${ }^{31} 114-115$ $\left.{ }^{\circ} \mathrm{C}\right)$; IR $\left(\mathrm{KBr}, \mathrm{cm}^{-1}\right): 2942,2127,1587,1496,1459,1330,1249,1105 ;{ }^{1} \mathrm{H}$ NMR (300 MHz, $\left.\mathrm{CDCl}_{3}\right): 2.69(\mathrm{t}, \mathrm{J}=2.4 \mathrm{~Hz}, 1 \mathrm{H}), 4.92(\mathrm{~d}, \mathrm{~J}=2.4 \mathrm{~Hz}, 2 \mathrm{H}), 7.36(\mathrm{~d}, \mathrm{~J}=8 \mathrm{~Hz}, 2 \mathrm{H}), 8.39(\mathrm{~d}, \mathrm{~J}=$ $8 \mathrm{~Hz}, 2 \mathrm{H}) ; \mathrm{MS}(\mathrm{m} / \mathrm{z}): 177\left[\mathrm{M}^{+}\right]$.

Synthesis 2-prop-2-ynyloxy-benzaldehyde (4). Yield: $84 \%$; mp $72{ }^{\circ} \mathrm{C}\left(\right.$ lit. $\left.^{32} 71{ }^{\circ} \mathrm{C}\right)$; IR (KBr, $\mathrm{cm}^{-1}$ ): 2925, 2124, 1681, 1484, 1399, 1287, 1235, 1189, 1105; ${ }^{1} \mathrm{H}$ NMR (300 MHz, $\left.\mathrm{CDCl}_{3}\right): 2.62$ 
$(\mathrm{t}, \mathrm{J}=2.4 \mathrm{~Hz}, 1 \mathrm{H}), 4.84(\mathrm{~d}, \mathrm{~J}=2.4 \mathrm{~Hz}, 2 \mathrm{H}), 7.82(\mathrm{~d}, 1 \mathrm{H}), 7.61(\mathrm{~d}, 1 \mathrm{H}), 7.21-7.26(\mathrm{~m}, 2 \mathrm{H})$; MS $(\mathrm{m} / \mathrm{z}): 160\left[\mathrm{M}^{+}\right]$.

Synthesis of 2-methyl-5-prop-2-ynyloxy-pyridine (5). Yield: 68\%; IR (KBr, cm$\left.{ }^{-1}\right)$ : 3025, 2926, 2120, 1574, 1495, 1455, 1389, 1275, 1025; ${ }^{1} \mathrm{H}$ NMR (300 MHz, $\left.\mathrm{CDCl}_{3}\right): 2.61$ (s, 3H), 2.69 (t, J $=2.4 \mathrm{~Hz}, 1 \mathrm{H}), 4.80(\mathrm{~d}, \mathrm{~J}=2.4 \mathrm{~Hz}, 2 \mathrm{H}), 7.16-7.21(\mathrm{~m}, 2 \mathrm{H}), 8.37(\mathrm{~s}, 1 \mathrm{H}) ; \mathrm{MS}(\mathrm{m} / \mathrm{z}): 147\left[\mathrm{M}^{+}\right]$.

Synthesis of 2-prop-2-ynyloxymethyl-furan ${ }^{35}$ (6). Yield: 65\%; ${ }^{1} \mathrm{H}$ NMR $\left(300 \mathrm{MHz}, \mathrm{CDCl}_{3}\right)$ : $2.56(\mathrm{t}, \mathrm{J}=2.4 \mathrm{~Hz}, 1 \mathrm{H}), 4.25(\mathrm{~s}, 2 \mathrm{H}), 4.67(\mathrm{~d}, \mathrm{~J}=2.4 \mathrm{~Hz}, 2 \mathrm{H}), 6.49$ (m, 2H), 7.55 (s, 1H); MS (m/z): $136\left[\mathrm{M}^{+}\right]$; Anal. calcd. for $\mathrm{C}_{8} \mathrm{H}_{8} \mathrm{O}_{2}$ : C, 70.57; H, 5.92. Found: C, 70.77; H, 6.15.

Synthesis of prop-2-ynylsulfanyl-benezene (7). Yield: 78\%; IR (KBr, cm $\left.{ }^{-1}\right): 3059,2922,2106$, 1585, 1480, 1438, 1233, 1025; ${ }^{1} \mathrm{H}$ NMR (300 MHz, $\left.\mathrm{CDCl}_{3}\right): 2.34(\mathrm{t}, \mathrm{J}=2.4 \mathrm{~Hz}, 1 \mathrm{H}), 3.50(\mathrm{~d}, \mathrm{~J}=$ $2.4 \mathrm{~Hz}, 2 \mathrm{H}), 7.10-7.22(\mathrm{~m}, 5 \mathrm{H})$; $\mathrm{MS}(\mathrm{m} / \mathrm{z}): 148\left[\mathrm{M}^{+}\right]$.

Synthesis of 2-prop-2-ynyloxy-tetrahydropyran (8). Yield: 83\%; ${ }^{1} \mathrm{H} \mathrm{NMR}\left(300 \mathrm{MHz}, \mathrm{CDCl}_{3}\right)$ : 1.47-1.75 (m, 6H), $2.37(\mathrm{t}, \mathrm{J}=2.4 \mathrm{~Hz}, 1 \mathrm{H}), 3.46(\mathrm{~m}, 1 \mathrm{H}), 3.76(\mathrm{~m}, 1 \mathrm{H}), 4.11(\mathrm{~d}, \mathrm{~J}=2.4 \mathrm{~Hz}, 2 \mathrm{H})$, $4.74(\mathrm{~m}, 1 \mathrm{H}) ; \operatorname{MS}(\mathrm{m} / \mathrm{z}): 140\left[\mathrm{M}^{+}\right]$.

Synthesis of chloro-(pent-4-en-2-ynyloxy)-benzene (9). Yield: $62 \%$; IR ( $\left.\mathrm{KBr}, \mathrm{cm}^{-1}\right)$ : 2911 , 2862, 2125, 1596, 1493, 1215, 1032; ${ }^{1} \mathrm{H}$ NMR (300 MHz, $\left.\mathrm{CDCl}_{3}\right)$ : 4.92 (s, 2H), 5.91 (d, 1H), 6.41 (d, 1H), 7.08-7.23 (m, 5H); MS (m/z): $192\left[\mathrm{M}^{+}\right], 194\left[\mathrm{M}^{+}+2\right]$.

Synthesis of chloro-(pent-4-en-2-ynyloxy)-tetrahydro-pyran ${ }^{29}$ (10). In a closed screw caped reaction vessel mixture of $\mathrm{Pd}\left(\mathrm{PPh}_{3}\right)_{4}(248 \mathrm{mg}, 0.22 \mathrm{mmol}), \mathrm{CuI}(272 \mathrm{mg}, 1.42 \mathrm{mmol}), n$ butylamine (2.6 gm, $35.46 \mathrm{mmol})$, 2-prop-2-ynyloxy-tetrahydro-pyran (1.0 gm, $7.14 \mathrm{mmol})$ and cis-dichloroethylene $(690 \mathrm{mg}, 7.1 \mathrm{mmol})$ in $5 \mathrm{~mL}$ of anhydrous benzene was irradiated in a microwave $(650 \mathrm{~W})$ for 3 minutes in a successive interval of 30 seconds each. Progress of the reaction was monitored by TLC and reaction completes in 3 minutes. Excess of solvent from the reaction mixture was removed under reduced pressure and the crude product thus obtained was purified by column chromatography (15\% ethyl acetate/hexane). Yield of compound 10, $870 \mathrm{mg}$ $(60.8 \%)$, along with compound $\mathbf{1 2}^{28}$ in $11 \%$ yield. Compound $\mathbf{1 0}^{29}$ : ${ }^{1} \mathrm{H}$ NMR (300 MHz, $\left.\mathrm{CDCl}_{3}\right): 1.35-1.74(\mathrm{~m}, 6 \mathrm{H}), 3.48(\mathrm{~m}, 1 \mathrm{H}), 3.78(\mathrm{~m}, 1 \mathrm{H}), 4.37(\mathrm{~s}, 2 \mathrm{H}), 4.79(\mathrm{~m}, 1 \mathrm{H}), 5.82(\mathrm{~d}, \mathrm{~J}=$ $7.4 \mathrm{~Hz}, 1 \mathrm{H}), 6.34(\mathrm{~d}, \mathrm{~J}=7.4 \mathrm{~Hz}, 1 \mathrm{H})$; MS-ESI $(\mathrm{m} / \mathrm{z}): 223.2810\left[\mathrm{M}^{+}+\mathrm{Na}\right]$; Anal. calcd. for $\mathrm{C}_{10} \mathrm{H}_{13} \mathrm{ClO}_{2}$ : C, 59.86; H, 6.53. Found: C, 60.12; H, 6.71.

Synthesis of 2-(8-phenoxy-oct-4-ene-2,6-diynyl-oxy)-tetrahydro-pyran (13). General procedure

The mixture of $\mathrm{Pd}\left(\mathrm{PPh}_{3}\right)_{4}(172 \mathrm{mg}, 0.15 \mathrm{mmol}), \mathrm{CuI}(113 \mathrm{mg}, 0.59 \mathrm{mmol}), n$-butylamine (1.10 gm, $15.04 \mathrm{mmol}), 2$-(5-chloro-pent-4-en-2-ynyloxy)-tetrahydropyran $\quad(\mathbf{1 0})^{29}(600 \mathrm{mg}, 2.99$ $\mathrm{mmol})$ and prop-2-ynyloxy-benzene (1) $(435 \mathrm{mg}, 3.3 \mathrm{mmol})$ in $5 \mathrm{~mL}$ of anhydrous benzene in a screw caped reaction vessel was irradiated in a microwave $(650 \mathrm{~W})$ for 4 minutes in a successive interval of 30 seconds each. The progress of the reaction was monitored by TLC. The reaction was completed in 4 minutes. Excess of solvent from the reaction mixture was removed under reduced pressure and crude product was purified by column chromatography (5\% ethyl acetate/hexane). Yield: $550 \mathrm{mg}(61.9 \%)$; DSC: $145^{\circ} \mathrm{C}$; IR $\left(\mathrm{KBr}, \mathrm{cm}^{-1}\right): 2945,2211,1597,1494$, 1364, 1262, 1214, 1120, 1077, 1024; ${ }^{1} \mathrm{H}$ NMR (300 MHz, $\left.\mathrm{CDCl}_{3}\right): 1.52-1.82(\mathrm{~m}, 6 \mathrm{H}), 3.53(\mathrm{~m}$, 
1H), $3.85(\mathrm{~m}, 1 \mathrm{H}), 4.40(\mathrm{~s}, 2 \mathrm{H}), 4.85-4.87(\mathrm{~m}, 3 \mathrm{H}), 5.86(\mathrm{~m}, 2 \mathrm{H}), 6.91-7.08(\mathrm{~m}, 3 \mathrm{H}), 7.29-7.32$ $(2 \mathrm{H}) ;{ }^{13} \mathrm{C}$ NMR $\left(75.5 \mathrm{MHz}, \mathrm{CDCl}_{3}\right): 19.24,25.22,30.42,54.58,56.38,61.89,82.88,84.06$, 91.46, 93.12, 96.61, 114.83, 120.00, 129.34, 155.96; MS-ESI (m/z): $319.4180\left[\mathrm{M}^{+}+\mathrm{Na}\right]$; Anal. calcd. for $\mathrm{C}_{19} \mathrm{H}_{20} \mathrm{O}_{3}$ : C, 77.00; H, 6.80. Found: C, 77.21; H, 6.99.

Synthesis of 1,8-bis(tetrahydropyran-2-yloxy)oct-4-ene-2,6-diyne ${ }^{28}$ (14). Yield: 59\%; IR $\left(\mathrm{KBr}, \mathrm{cm}^{-1}\right): 2942,2870,2210,1440,1389,1344,1201,1119,1078,1023 ;{ }^{1} \mathrm{H}$ NMR (300 MHz, $\left.\mathrm{CDCl}_{3}\right): 1.42-1.85(\mathrm{~m}, 12 \mathrm{H}), 3.54(\mathrm{~m} 2 \mathrm{H}), 3.85(\mathrm{~m}, 2 \mathrm{H}), 4.45(\mathrm{~s}, 4 \mathrm{H}), 4.78(\mathrm{~s} 2 \mathrm{H}), 5.85(\mathrm{~s} 2 \mathrm{H})$; ${ }^{13} \mathrm{C}$ NMR (75.5 MHz): 18.6, 25.1, 30.0, 54.3, 61.6, 82.6, 92.6, 96.3, 119.0; MS (m/z) $304\left[\mathrm{M}^{+}\right]$.

Synthesis of 2-(8-naphthalen-2-yloxy)-oct-4-ene-2,6-diynyloxy)-tetrahydro-pyran (15). Yield: 54\%; DSC: $153{ }^{\circ} \mathrm{C}$; IR $\left(\mathrm{KBr}, \mathrm{cm}^{-1}\right): 2944,2868,2211,1629,1600,1510,1469,1256$, 1175, 1119; ${ }^{1} \mathrm{H}$ NMR (300 MHz, $\left.\mathrm{CDCl}_{3}\right): 1.51-1.79(\mathrm{~m}, 6 \mathrm{H}), 3.53$ (m, 1H), $3.78(\mathrm{~m}, 1 \mathrm{H}), 4.32$ $(\mathrm{s}, 2 \mathrm{H}), 4.52(\mathrm{~s}, 2 \mathrm{H}), 4.83(\mathrm{~m}, 1 \mathrm{H}), 4.99(\mathrm{~s}, 2 \mathrm{H}), 7.17-7.37(\mathrm{~m}, 5 \mathrm{H}), 7.72-7.76(\mathrm{~m}, 2 \mathrm{H})$; MS (m/z): $346\left[\mathrm{M}^{+}\right]$; Anal. calcd. for $\mathrm{C}_{23} \mathrm{H}_{22} \mathrm{O}_{3}$ : C, 79.74; H, 6.40. Found: C, 79.51; H, 6.69.

Synthesis of 2-[8-(tetrahydropyran-2-yloxy)-oct -4-ene-2,6-diynyloxy)-benzaldehyde (16). Yield : 52\%; DSC: $142{ }^{\circ} \mathrm{C}$; IR (KBr, cm $\left.{ }^{-1}\right)$ : 2972, 2856, 2212, 1610, 1622, 1599, 1481, 1458, 1287, 1218, 1012; ${ }^{1} \mathrm{HNMR}\left(300 \mathrm{MHz}, \mathrm{CDCl}_{3}\right)$ : 1.47-1.76 (m, 6H), $3.44(\mathrm{~m}, 1 \mathrm{H}), 3.76(\mathrm{~m}, 1 \mathrm{H})$, $4.31(\mathrm{~s}, 2 \mathrm{H}), 4.78(\mathrm{~s}, 1 \mathrm{H}), 4.95(\mathrm{~s}, 2 \mathrm{H}), 5.82(\mathrm{~m}, 2 \mathrm{H}), 6.98-7.11(\mathrm{~m}, 2 \mathrm{H}), 7.47-7.52(\mathrm{~m}, 1 \mathrm{H}), 7.66$ $(\mathrm{m}, 1 \mathrm{H}), 10.41(\mathrm{~s}, 1 \mathrm{H})$; MS (m/z): $324\left[\mathrm{M}^{+}\right]$; Anal. calcd. for $\mathrm{C}_{20} \mathrm{H}_{20} \mathrm{O}_{4}: \mathrm{C}, 74.06 ; \mathrm{H}, 6.21$. Found: C, 74.21; H, 6.08.

Synthesis of 2-methyl-5-[tetrahydro-pyran-2-yloxy)-oct-4-ene-2,6-diynyloxy)-pyridine (17). Yield: 64\%; DSC: $146{ }^{\circ} \mathrm{C}$; IR ( $\left.\mathrm{KBr}, \mathrm{cm}^{-1}\right): 2943,2868,2211,1573,1489,1453,1389,1270$, 1121, 1023; ${ }^{1} \mathrm{H}$ NMR (300 MHz, $\left.\mathrm{CDCl}_{3}\right): 1.52-1.77$ (m, 6H), $2.51(\mathrm{~s}, 3 \mathrm{H}), 3.55(\mathrm{~m}, 1 \mathrm{H}), 3.82$ $(\mathrm{m}, 1 \mathrm{H}), 4.42(\mathrm{~s}, 2 \mathrm{H}), 4.88(\mathrm{~s}, 1 \mathrm{H}), 4.91(\mathrm{~s}, 2 \mathrm{H}), 5.88(\mathrm{~m}, 2 \mathrm{H}), 7.28-7.30(\mathrm{~m}, 2 \mathrm{H}), 8.15(\mathrm{~m}, 1 \mathrm{H})$; MS (m/z): $312\left[\mathrm{M}^{+}+\mathrm{H}\right], 279,228$; Anal. calcd. for $\mathrm{C}_{19} \mathrm{H}_{21} \mathrm{NO}_{3}$ : C, 73.29; H, 6.80. Found: C, $73.55 ; \mathrm{H}, 6.59$.

Synthesis of trimethyl-[7-(tetrahydro-pyran-2-yloxy)-hept-3-ene-1,5-diynyl]-silane ${ }^{36}$ (18). Yield: 66\%; DSC: $171{ }^{\circ} \mathrm{C}$; IR $\left(\mathrm{KBr}, \mathrm{cm}^{-1}\right)$ : 2953, 2872, 2145, 1251, 1120, 1027; ${ }^{1} \mathrm{H}$ NMR (300 $\left.\mathrm{MHz}, \mathrm{CDCl}_{3}\right): 0.22(\mathrm{~s}, 9 \mathrm{H}), 1.48-1.75(\mathrm{~m}, 6 \mathrm{H}), 3.46(\mathrm{~m}, 1 \mathrm{H}), 3.82(\mathrm{~m}, 1 \mathrm{H}), 4.46(\mathrm{~s}, 2 \mathrm{H}), 4.81(\mathrm{~s}$, $1 \mathrm{H}), 5.78(\mathrm{~s}, 2 \mathrm{H}) ; \mathrm{MS}(\mathrm{m} / \mathrm{z}): 262\left[\mathrm{M}^{+}\right]$; Anal. calcd. for $\mathrm{C}_{15} \mathrm{H}_{22} \mathrm{O}_{2} \mathrm{Si}: \mathrm{C}, 68.65 ; \mathrm{H}, 8.45$. Found: $\mathrm{C}$, $68.85 ; \mathrm{H}, 8.78$.

Synthesis of 2-[8-(furan-2-ylmethoxy)-oct-4-ene-2,6-diynyloxy]-tetrahydro-pyran (19). Yield: 56\%; IR (KBr, cm $\left.{ }^{-1}\right)$ : 2943, 2853, 2211, 1503, 1440, 1348, 1202, 1152, 1120, 1069, 1023; ${ }^{1} \mathrm{H}$ NMR (300 MHz, $\left.\mathrm{CDCl}_{3}\right)$ : 1.50-1.81 (m, 6H), $3.50(\mathrm{~m}, 1 \mathrm{H}), 3.82(\mathrm{~m}, 1 \mathrm{H}), 4.35(\mathrm{~s}, 2 \mathrm{H}), 4.45$ $(\mathrm{d}, 2 \mathrm{H}), 4.61(\mathrm{~s}, 2 \mathrm{H}), 4.86(\mathrm{~m}, 1 \mathrm{H}), 5.88(\mathrm{~s}, 2 \mathrm{H}), 6.40(\mathrm{~m}, 2 \mathrm{H}), 7.42(\mathrm{~d}, 1 \mathrm{H}) ; \mathrm{MS}(\mathrm{m} / \mathrm{z}): 300$ $\left[\mathrm{M}^{+}\right]$; Anal. calcd. for $\mathrm{C}_{18} \mathrm{H}_{20} \mathrm{O}_{4}: \mathrm{C}, 71.98 ; \mathrm{H}, 6.71$. Found: $\mathrm{C}, 72.21 ; \mathrm{H}, 6.89$.

Synthesis of 1,1 '-[(4Z)-oct-4-ene-2,6-diyne-1,8-diylbis(oxy)dibenzene (20). Yield: 63\%; DSC: $151{ }^{\circ} \mathrm{C}$; ${ }^{1} \mathrm{H}$ NMR $\left(300 \mathrm{MHz}, \mathrm{CDCl}_{3}\right): 4.78$ (s, 4H), $5.86(\mathrm{~s}, 2 \mathrm{H}), 6.99(\mathrm{~m}, 6 \mathrm{H}), 7.29$ (m, 4H); MS-ESI (m/z): $311.2951\left[\mathrm{M}^{+}+\mathrm{Na}\right]$; Anal. calcd. for $\mathrm{C}_{20} \mathrm{H}_{16} \mathrm{O}_{2}: \mathrm{C}, 83.31 ; \mathrm{H}, 5.59$. Found: C, 83.28; H, 5.89. 
Synthesis of 2-(8-Phenoxy-oct-4-ene-2,6- diynyloxy)-benzaldehyde (21). Yield: 52\%; DSC: $152{ }^{\circ} \mathrm{C}$; IR (KBr, cm $\left.{ }^{-1}\right): 2952,2864,2202,1689,1622,1598,1483,1288,1216,1031 ;{ }^{1} \mathrm{H} \mathrm{NMR}$ $\left(300 \mathrm{MHz}, \mathrm{CDCl}_{3}\right): 4.79(\mathrm{~s}, 2 \mathrm{H}), 4.88(\mathrm{~s}, 2 \mathrm{H}), 5.86(\mathrm{~s}, 2 \mathrm{H}), 6.96-7.11(\mathrm{~m}, 5 \mathrm{H}), 7.24-7.30(\mathrm{~m}$, $2 \mathrm{H}), 7.58(\mathrm{~m}, 1 \mathrm{H}), 7.86(\mathrm{~m}, 1 \mathrm{H}), 10.48(\mathrm{~s}, 1 \mathrm{H}) ; \mathrm{MS}(\mathrm{m} / \mathrm{z}): 316\left[\mathrm{M}^{+}\right]$; Anal. calcd. for $\mathrm{C}_{21} \mathrm{H}_{16} \mathrm{O}_{3}$ : C, 79.73; H, 5.10. Found: C, 79.49; H, 5.29.

Synthesis of 2-methyl-5-(8-phenoxy-oct-4-ene-2,6-diynyloxy)-pyridine (22). Yield: 62\%; DSC: $139{ }^{\circ} \mathrm{C}$; IR (KBr, cm $\left.{ }^{-1}\right): 2922,2863,2211,1598,1494,1370,1264,1213,1094,1030 ;{ }^{1} \mathrm{H}$ NMR (300 MHz, $\left.\mathrm{CDCl}_{3}\right): 2.48(\mathrm{~s}, 3 \mathrm{H}), 4.77(\mathrm{~s}, 2 \mathrm{H}), 4.84(\mathrm{~s}, 2 \mathrm{H}), 5.92(\mathrm{~m}, 2 \mathrm{H}), 7.11-7.23(\mathrm{~m}$, $4 \mathrm{H}), 7.28-7.34(\mathrm{~m}, 3 \mathrm{H}), 8.28(\mathrm{~s}, 1 \mathrm{H})$; MS-ESI $(\mathrm{m} / \mathrm{z}): 304\left[\mathrm{M}^{+}+\mathrm{H}\right], 279$; Anal. calcd. for $\mathrm{C}_{20} \mathrm{H}_{17} \mathrm{NO}_{2}$ : C, 79.19; H, 5.65; N, 4.62. Found: C, 79.41; H, 5.76; N, 4.83.

Synthesis of 2-(8-phenoxy-oct-4-ene-2,6-diynyloxymethyl)-furan (23). Yield: 59\%; DSC: 141 ${ }^{\circ} \mathrm{C}$; IR (KBr, cm $\left.{ }^{-1}\right): 2912,2856,2211,1598,1494,1350,1261,1214,1151,1068,1031 ;{ }^{1} \mathrm{H}$ NMR (300 MHz, $\left.\mathrm{CDCl}_{3}\right): 4.22(\mathrm{~s}, 2 \mathrm{H}), 4.56(\mathrm{~s}, 2 \mathrm{H}), 4.85(\mathrm{~s}, 2 \mathrm{H}), 5.87(\mathrm{~s}, 2 \mathrm{H}), 6.36(\mathrm{~m}, 2 \mathrm{H})$, $6.95(\mathrm{~m}, 4 \mathrm{H}), 7.28-7.39(\mathrm{~m}, 2 \mathrm{H})$; MS (m/z): $293\left[\mathrm{M}^{+}+\mathrm{H}\right], 279,225,161$; Anal. calcd. for $\mathrm{C}_{19} \mathrm{H}_{16} \mathrm{O}_{3}$ : C, 78.06; H, 5.52. Found: C, 78.27; H, 5.74.

Synthesis of trimethyl-(7-phenoxy-hept-3-ene-1,5-diynyl)-silane (24). Yield: 65\%; DSC: 175 ${ }^{\circ} \mathrm{C}$; IR (KBr, cm $\left.{ }^{-1}\right): 2960,2901,2143,1598,1494,1370,1214,1032 ;{ }^{1} \mathrm{H}$ NMR (300 MHz, $\left.\mathrm{CDCl}_{3}\right): 0.21(\mathrm{~s}, 9 \mathrm{H}), 4.88(\mathrm{~s}, 2 \mathrm{H}), 5.85(\mathrm{~s}, 2 \mathrm{H}), 6.98-7.01(\mathrm{~m}, 3 \mathrm{H}), 7.29(\mathrm{~m}, 2 \mathrm{H}) ; \mathrm{MS}(\mathrm{m} / \mathrm{z}): 254$ $\left[\mathrm{M}^{+}\right]$; Anal. calcd. for $\mathrm{C}_{16} \mathrm{H}_{18} \mathrm{OSi}$ : C, 75.54; H, 7.13. Found: C, 75.73; H, 7.23.

\section{Synthesis of 1,2'-[thiene-2,3-diylbis(prop-1-yne-1,3-diyloxy)]bis-tetrahydro-2H-pyran (26)}

The mixture of $\mathrm{Pd}\left(\mathrm{PPh}_{3}\right)_{4}(248 \mathrm{mg}, 0.22 \mathrm{mmol})$, CuI (272 mg, $\left.1.42 \mathrm{mmol}\right), n$-butylamine (2.6 gm, $35.46 \mathrm{mmol})$, 2-prop-2-ynyloxy-tetrahydro-pyran or 2-methyl-5-prop-2-ynyloxy-pyridine (7.14 mmol) and 2,3-dibromothiophene $(690 \mathrm{mg}, 7.1 \mathrm{mmol})$ in $5 \mathrm{~mL}$ of anhydrous benzene was irradiated in a closed screw caped reaction vessel in microwave at $650 \mathrm{~W}$ for 3 minutes in a successive intervals of 30 seconds each. Progress of the reaction was monitored by TLC and after 3 minutes reaction was complete. Excess of solvent from the reaction mixture was removed under reduced pressure and the crude product thus obtained was purified by column chromatography (15\% ethyl acetate/hexane). Yield: 52\%; DSC: $230{ }^{\circ} \mathrm{C}$; IR $\left(\mathrm{KBr}, \mathrm{cm}^{-1}\right): 2943$, 2870, 2225, 1507, 1440, 1348, 1201, 1120, 1078; ${ }^{1} \mathrm{H}$ NMR (300 MHz, $\left.\mathrm{CDCl}_{3}\right)$ : 1.46-1.76 (m, $12 \mathrm{H}), 3.51(\mathrm{~m}, 2 \mathrm{H}), 3.77(\mathrm{~m}, 2 \mathrm{H}), 4.43(\mathrm{~s}, 4 \mathrm{H}), 4.86(\mathrm{~m}, 2 \mathrm{H}), 6.86(\mathrm{~d}, \mathrm{~J}=6 \mathrm{~Hz}, 1 \mathrm{H}), 7.12(\mathrm{~d}, \mathrm{~J}=$ $6 \mathrm{~Hz}, 1 \mathrm{H}) ;{ }^{13} \mathrm{C} \mathrm{NMR}\left(75.5 \mathrm{MHz}, \mathrm{CDCl}_{3}\right): 19.23,24.92,29.77,54.24,61.51,77.88,92.45,93.98$, 96.31, 126.66, 126.82, 129.45, 129.68; MS (m/z): $361\left[\mathrm{M}^{+}+\mathrm{H}\right]$; Anal. calcd. for $\mathrm{C}_{20} \mathrm{H}_{24} \mathrm{O}_{4} \mathrm{~S}: \mathrm{C}$, 66.64; H, 6.71. Found: C, 66.93; H, 6.38.

Synthesis of 3,3' [thiene-2,3-diylbis(prop-1-yne-1,3-diyloxy)]bis(6-methylpyridine) (27). As discussed for the synthesis of compound 26. Yield: 54\%; DSC: $195{ }^{\circ} \mathrm{C}$; ${ }^{1} \mathrm{H} \mathrm{NMR}(300 \mathrm{MHz}$, $\left.\mathrm{CDCl}_{3}\right): 2.65(\mathrm{~s}, 6 \mathrm{H}), 5.11(\mathrm{~s}, 4 \mathrm{H}), 6.94(\mathrm{~d}, \mathrm{~J}=6 \mathrm{~Hz}, 1 \mathrm{H}), 7.08(\mathrm{~d}, \mathrm{~J}=6 \mathrm{~Hz}, 1 \mathrm{H}), 7.25-7.33(\mathrm{~m}$, $4 \mathrm{H}), 8.25(\mathrm{~m}, 2 \mathrm{H}) ; \mathrm{MS}(\mathrm{m} / \mathrm{z}): 374\left[\mathrm{M}^{+}\right], 376\left[\mathrm{M}^{+}+2\right]$; Anal. calcd. for $\mathrm{C}_{22} \mathrm{H}_{18} \mathrm{~N}_{2} \mathrm{O}_{2} \mathrm{~S}: \mathrm{C}, 70.57 ; \mathrm{H}$, 4.85; N, 7.48. Found: C, 70.41; H, 4.48; N, 7.66. 


\section{Acknowledgements}

DSR thanks Department of Science and Technology, New Delhi, India for financial support (DO No: SR/FTP/CSA-18/2003) and NMR, MS (DST-FIST), IR facility, Department of Chemistry, University Science Instrumentation Center, University of Delhi and Dr. S. K. Dubey, Jubilant Organosis for helping us to get the analytical data.

\section{References}

1. Smith, A. L.; Nicolaou, K. C. J. Med. Chem. 1996, 39, 2103.

2. Rawat, D. S.; Zaleski, J. M. Synlett 2004, 392.

3. Zaleski, J. M.; Rawat, D. S. US Patent 2006, 6987132 B1.

4. Shen, B.; Liu, W.; Nonaka, K. Curr. Med. Chem. 2003, 10, 2317.

5. Nicolaou, K. C.; Smith, A. L. Acc. Chem. Res. 1992, 25, 497.

6. Suzuki, I.; Shigenaga, A.; Nemoto, H.; Shibuya, M. Tetrahedron Lett. 2004, 45, 1955.

7. Dai, W. -M. Curr. Med. Chem. 2003, 10, 2265.

8. Wang, Z.; He, Q.; Liang, Y.; Wang, D.; Li, Yi-Y.; Li, D. Biochem. Pharm. 2003, 65, 1767.

9. Lin, C. -F.; Lo, Y. -H.; Hsieh, M. -C.; Chen, Y.- H.; Wanga, J. -J.; Wua, M. -J. Bioorg. Med. Chem. 2005, 13, 3565.

10. Fouad, F. S.; Wright, J. M.; Plourde, G.; Purohit, A. D.; Wyatt, J. K.; El-Shafey, A.; Hynd, G.; Crasto, C. F.; Lin, Y.; Jones, G. B. J. Org. Chem. 2005, 70, 9789.

11. Lin, C. -F.; Hsieh, P. -C.; Lu, W. -D.; Chiub H. -F.; Wua, M. -J. Bioorg. Med. Chem. 2001, 9, 1707.

12. Nicolaou, K. C.; Dia, W.-M. Angew. Chem. Int Ed.. 1991, 30, 1387.

13. Benites, P. J.; Holmberg, R. C.; Rawat, D. S.; Kraft, B. J.; Klein, L. J.; Peters, D. G.; Thorp, H. H.; Zaleski, J. M. J. Am. Chem. Soc. 2003, 125, 6434.

14. Basak, A.; Bag, S. S.; Basak, A. Bioorg. Med. Chem. 2005, 13, 4096.

15. Banfi, L.; Basso, A.; Guanti, G.; Riva, R. Arkivoc 2006, (vii), 261.

16. Rawat, D. S.; Zaleski, J. M. J. Am. Chem. Soc. 2001, 123, 9675.

17. Benites, P. J.; Rawat, D. S.; Zaleski, J. M. J. Am. Chem. Soc. 2000, 122, 7208.

18. Kovalenko, S. V.; Alabugin, I. V. Chem. Commun. 2005, 1444.

19. Jones, G. B.; Fouad, F. S. Curr. Pharm. Des. 2002, 8, 2415.

20. Sonogashira, K.; Tohda, Y.; Hagihara, N. Tetrahedron Lett. 1975, 16, 4467.

21. Ratovelomanana, V.; Linstrumelle, G. Tetrahedron Lett. 1981, 22, 315.

22. Vollhardt, K. P. C.; Winn. L. S. Tetrahedron Lett. 1985, 26, 709.

23. Wannberg, J.; Sabnis, Y. A.; Vrang, L.; Samuelsson, B.; Karlén, A.; Hallberg, A.; Larhed, M. Bioorg. Med. Chem. 2006, 14, 5303.

24. Miljanic, O. S.; Vollhardt, K. P. C.; Whitener, G. D. Synlett 2003, 29.

25. He, H.; Wu, Y. J. Tetrahedron Lett. 2004, 45, 3237. 
26. Kappe, C. O. Angew. Chem. Int. Ed. 2004, 43, 6250.

27. Burdon, J.; Coe, P. L.; Marsh, C. R.; Tatlow, J. C. Chem. Commun. 1967, 1259.

28. Konig, B.; Pitsch, W.; Dix, I.; Jones, P. G. Synthesis 1996, 446.

29. Ratovelomanana, V.; Linstrumelle, G. Tetrahedron Lett. 1984, 25, 6001.

30. Pal, M.; Parasuraman, K.; Yeleswarapu, K. R. Org. Lett. 2003, 5, 349.

31. Agag, T.; Takeichi, T. Macromolecules 2001, 34, 7257.

32. Booth, S. E.; Jenkins, P. R.; Swain, C. J.; Sweeney, J. B. J. Chem. Soc., Perkin Trans. 1, 1994, 3499.

33. Martín-Matute, B.; Nevado, C.; Cárdenas, D. J.; Echavarren, A. M. J. Am. Chem. Soc. 2003, 125, 5757.

34. Kundu, N. G.; Nandi, B. Tetrahedron 2001, 57, 5885.

35. Kim, S.; Lawson, J. A.; Pratico, D.; FitzGeraldb, G. A.; Rokacha, J. Tetrahedron Lett. 2002, 43, 2801.

36. Lin, C.-F; Wu, M.-J. J. Org. Chem. 1997, 62, 4546. 